\title{
MIP-based biomimetic sensor for the electronic detection of serotonin in human blood plasma
}

\author{
M. Peeters ${ }^{\mathrm{a}, *}$, F.J. Troost ${ }^{\mathrm{b}}$, B. van Grinsven ${ }^{\mathrm{a}}$, F. Horemans ${ }^{\mathrm{a}}$, J. Alenus ${ }^{\mathrm{a}}$, M.S. Murib ${ }^{\mathrm{a}}$, D. Keszthelyi ${ }^{\mathrm{b}}$, \\ A. Ethirajan ${ }^{a}$, R. Thoelen ${ }^{a, c}$, T.J. Cleij ${ }^{a}$, P. Wagner ${ }^{a, d}$ \\ a Institute for Materials Research IMO, Hasselt University, Wetenschapspark 1, B-3590 Diepenbeek, Belgium

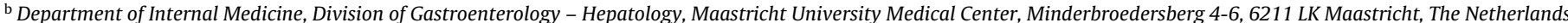

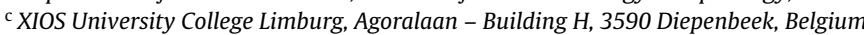 \\ d IMEC vzw, Division IMOMEC, Wetenschapspark 1, 3590 Diepenbeek, Belgium
}

\section{A R T I C L E I N F O}

\section{Article history:}

Received 14 March 2012

Received in revised form 8 May 2012

Accepted 8 May 2012

Available online 18 May 2012

\section{Keywords:}

Biomimetic sensors

Serotonin

Molecularly imprinted polymers

Impedance spectroscopy

Equivalent circuit modeling

\begin{abstract}
A B S T R A C T
Serotonin is an important signaling molecule in the human body. The detection of serotonin is commonly performed by high performance liquid chromatography (HPLC), which is costly and time consuming due to extensive sample preparation. We will show that these problems can be overcome by using molecularly imprinted polymers (MIPs) as synthetic receptors in combination with impedance spectroscopy as readout technique. The MIPs were prepared with several blends of the underlying monomers and the best performing MIP material was selected by optical batch-rebinding experiments. MIP microparticles were then integrated in an impedimetric sensor cell and dose-response curves were measured in PBS buffer and in non-diluted blood plasma. The sensor provides reliable data in the physiologically relevant concentration regime as an independent validation by HPLC measurements demonstrates. Finally, we show that the impedimetric response upon serotonin binding can be attributed to a capacitive effect at the interface between the MIP particles and the plasma.
\end{abstract}

(C) 2012 Elsevier B.V. All rights reserved.

\section{Introduction}

Serotonin (5-hydroxytryptamine, 5-HT) is a metabolite of the essential amino acid tryptophan and its role in smooth muscle contraction was already reported in 1951 [1]. The structure of serotonin, its natural metabolite 5-hydroxyindoleacetic acid (5-HIAA), and its oxidation product are shown in Fig. 1. Today, it is known that the serotoninergic system is steering numerous behavioral and physiological functions including emotions, sleep, and appetite [2]. Abnormalities of serotonin-related processes in the central nervous system can lead to severe mental disorders including anorexia, depression, and schizophrenia [2,3]. Also, anomalous serotonin levels are found in patients with hypertension [4], migraine, fibrotic syndrome, and carcinoid tumors [5,6]. Recently, it was recognized that serotonin is also involved in the regulation of the gastrointestinal functions and this insight has led to novel treatments for gastrointestinal disorders [7-9]. For diagnostic purposes, serotonin concentrations are usually analyzed in portal blood and in the systemic circulation, where plasma levels of 5-20 nM define the typical range for healthy individuals $[6,10]$.

\footnotetext{
* Corresponding author. Tel.: +32 11 268876; fax: +32 11268899 . E-mail address: marloes.peeters@uhasselt.be (M. Peeters).
}

Serotonin belongs to the class of indoles and this group of aromatic, heterocyclic molecules is sensitive to light, oxygen and changes in $\mathrm{pH}$. Therefore, special precautions must be taken to prevent oxidation during preparation and handling of patients' samples [11,12]. Established detection techniques such as solvent extraction and ion exchange chromatography require extensive sample preparation and are aspecific with respect to other indole species [13]. This can be overcome by high pressure liquid chromatography (HPLC), which can distinguish between different indoles. Therefore, it is currently the most common technique in the field $[11,14]$, but it is especially costly and requires sophisticated equipment, making it unsuitable for routine tests [2].

Other options include electrochemical techniques: For instance Sarada et al. were able to measure serotonin in the $10-1000 \mathrm{nM}$ range in aqueous media using amperometric detection [15]. Wu et al. developed carbon-nanotube coated glass electrodes suitable for cyclic voltammetry measurements in spiked human blood serum [16]. Using the same technique, Kumara Swamy and Venton attempted a first in vivo measurement in the striatum of an anesthetized rat [17]. Upon administration of the serotonin precursor 5-hydroxytryptophan, a change in the oxidation current was observed, however it was not possible to determine the exact concentration. Summarizing, with electrochemical techniques it is not possible so far to measure serotonin selectively in the relevant concentration range of 'real' biological samples. 

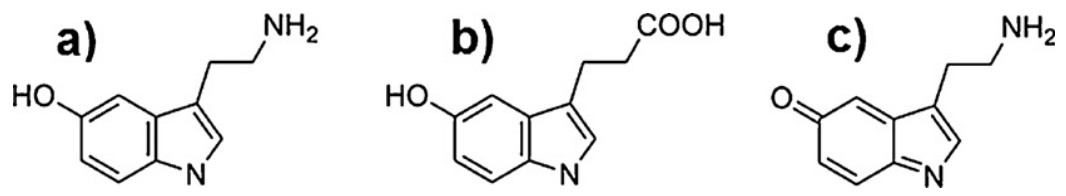

Fig. 1. The structure of serotonin (a), its metabolite 5-HIAA (b) and the chemical oxidation product (c).

An alternative route to serotonin detection can be found in molecular imprinting. Using molecularly imprinted polymers (MIPs) as synthetic receptors offers, in addition to specific recognition, several benefits: First, MIPs can be synthesized at a rather low cost via established polymer-chemical routes [18-20]. Second, MIPs can be stored for long time scales and maintain their receptor properties in wide ranges of temperature, $\mathrm{pH}$, and ionic strength [21-23]. Third, the recognition of target molecules is reversible for non-covalent imprinting, allowing MIP-based sensors to be used repetitively [24].

Literature reports on the synthesis of MIPs for serotonin recognition are sparse and very recent: Kitade et al. developed a MIP with methacrylic acid (MAA) as functional monomer [25]. Analysis with optical spectometry allowed detecting serotonin in water with a detection limit of $1 \mu \mathrm{M}$. Okutucu and Telefoncu also used methacrylic acid and packed the MIPs obtained by bulkpolymerization into separation columns. The analysis of plasma platelet samples spiked with $100 \mathrm{nM}$ of serotonin was performed with HPLC [26]. After optimization, Okutucu et al. detected spiked serotonin concentrations in the $\mu \mathrm{M}$ range also in human blood [27]. Khurshid et al. combined the charged monomer methacrylic acid with the neutral monomer methacrylamide to detect $1 \mathrm{mM}$ of serotonin in the dimethylformamide with a microplate reader [28].

While the synthesis of the MIPs is low-cost, the aforementioned detection techniques are still not suited for point-of-care applications. This can be solved by combining MIP receptors with electronic read-out strategies. In previous work, first examples were demonstrated with MIP-based impedimetric sensors for nicotine [29] and for histamine [30,31]. These sensors have detection limits of $2 \mathrm{nM}$ in buffer solutions, but the functioning in biological matrices was not yet demonstrated. Other electronic readout techniques for MIP-based sensors, such as voltammetry and quartz crystal microbalances, have also been reported, but the detectable concentration range is generally at the millimolar scale $[31,32]$. Since the serotonin level in human blood is typically $5-20 \mathrm{nM}$, impedance spectroscopy is therefore the method of choice. To our knowledge, there are no previous reports in literature combining the molecular imprinting of serotonin with impedimetric read-out.

The goal of this study was threefold: first, we aimed to optimize the monomer blends and MIP synthesis with respect to affinity and selectivity. Especially, the MIPs should allow discriminating between serotonin, its oxidized form and its metabolite 5-HIAA. Next, the goal was to develop an impedimetric sensor that is able to cope with the fast oxidization of serotonin and special care needs to be taken to circumvent non-specific responses from competing molecules in the complex matrix of blood plasma. To this end, non-imprinted polymers (NIPs) will be used as a reference channel. Finally, we aimed to set up equivalent-circuit modeling to assist in understanding the physical origin of the impedance increase, which is observed upon the molecular recognition of serotonin at the MIP-coated sensor electrodes.

\section{Materials and methods}

\subsection{Chemical reagents}

Ethylene glycol dimethacrylate (EGDM), methacrylic acid (MAA), acrylamide (AM) and dimethylsulfoxide (DMSO) were purchased from Acros (Geel, Belgium). Prior to polymerization, the stabilizers in the MAA and EGDM were removed by filtration over alumina. Azobusisobutyronitrile (AIBN) was purchased from Fluka (Buchs, Switzerland). The target molecule serotonin and its competitor, the metabolite 5-HIAA, were obtained from Acros (Geel, Belgium). All solvents were of analytical grade (Acros, Geel, Belgium) and used without further purification. The PPV derivative, $\mathrm{OC}_{1} \mathrm{C}_{10}$-PPV, which served as the immobilization layer on the impedimetric sensor electrodes, was synthesized via the sulfinyl precursor route [33]. All chemical and physical properties of this conjugated polymer were in agreement with previously reported data. For the impedance measurements a home-made $1 \times$ phosphate buffered saline (PBS) solution was used.

\subsection{MIP synthesis}

The MIP synthesis was optimized to achieve a high affinity and selectivity for serotonin. The combination of two functional monomers, methacrylic acid (MAA) and acrylamide (AM), was studied by varying the monomer ratios. Hereby, the following MAA:AM ratios were considered: $1: 0,1: 1,1: 3$, and $0: 1$. As a measure of the specificity, the imprint factor was selected, which corresponds to the amount of target molecules bound per gram of the MIP divided by that of the NIP. The imprint factors were determined by optical batch rebinding experiments at a free target concentration of $0.3 \mathrm{mM}$ using a Varian Cary 500 UV-vis-NIR spectrophotometer (Leuven, Belgium). Briefly summarized, MIPs composed solely of MAA or AM did not show specific binding properties. Upon mixing MAA and AM in a 1:1 ratio, an improved imprint factor of 1.4 was achieved. The highest imprint factor (3.64) was obtained with MIPs synthesized from the 1:3 blend. This MIP was prepared as follows: first, a mixture of MAA $(2.84 \mathrm{mmol})$, AM $(8.50 \mathrm{mmol})$, EGDM $(22.72 \mathrm{mmol})$ and AIBN $(0.61 \mathrm{mmol})$ was dissolved in $7 \mathrm{ml}$ DMSO together with the template molecule serotonin $(5.67 \mathrm{mmol})$. This solution was degassed with $\mathrm{N}_{2}$ and polymerized in a UV oven for $12 \mathrm{~h}$. After polymerization, the bulk polymer was ground and sieved to obtain microparticles with a size smaller than $25 \mu \mathrm{m}$. This was verified by SEM-images, which showed a similar particle size distribution for the MIP and NIP-particles. Finally, the serotonin was removed from the MIP powders by Soxhlet extraction with methanol ( $48 \mathrm{~h})$, a mixture of acetic acid/acetonitrile (1/1) (48 h) and again methanol (12 h). The extracted powders were dried in vacuum for $12 \mathrm{~h}$ at room temperature. A non-imprinted polymer (NIP) was synthesized accordingly, but without the presence of the target molecule. These MIP and NIP powders were used in all further batch-rebinding and impedimetric measurements.

\subsection{Preparation of blood-plasma samples and HPLC characterization}

Blood samples were obtained from three healthy volunteers, person $\mathrm{A}, \mathrm{B}$ and $\mathrm{C}$, and divided over $4 \mathrm{ml} \mathrm{K}_{2}$ EDTA tubes in order to prevent coagulation. The blood collection tubes also contained $0.1 \mathrm{ml}$ of ascorbic acid ( $1.4 \mathrm{~g}$ ascorbic acid $/ 100 \mathrm{ml}$ distilled water). This addition is necessary to prevent the conversion of serotonin to 5-HIAA by the enzyme monoamine oxidase, which normally occurs within several seconds. It was proven earlier that this 


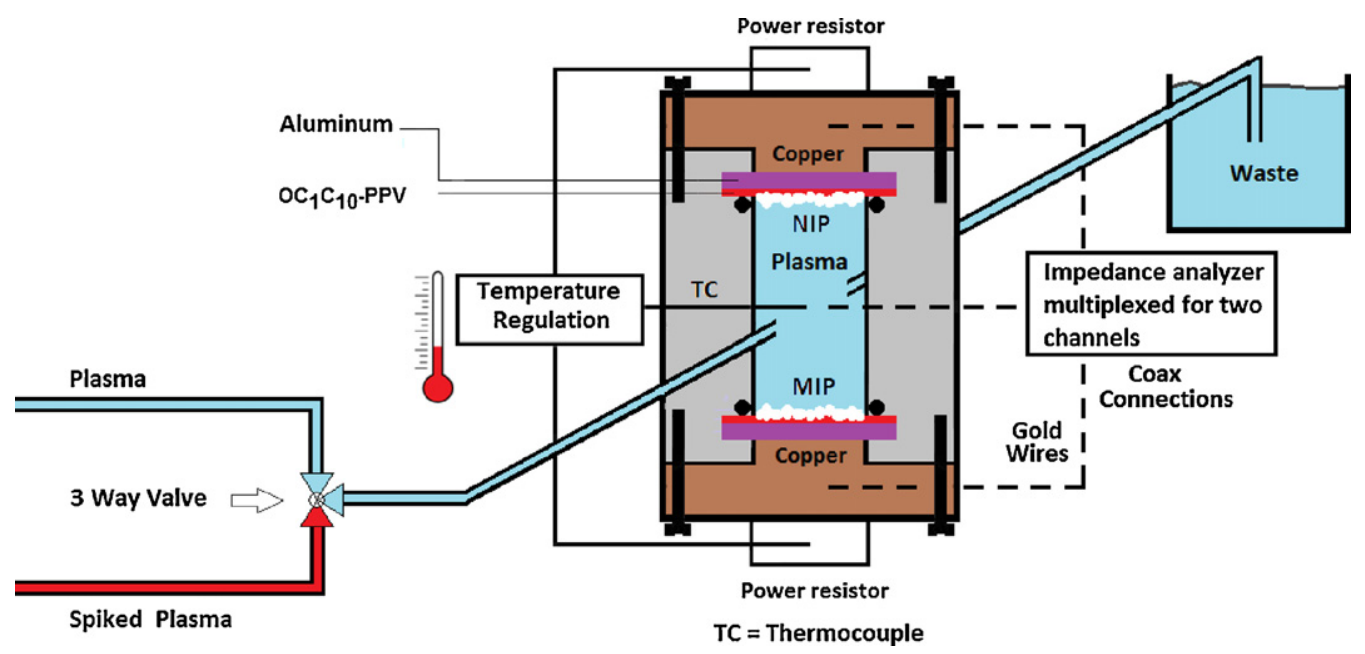

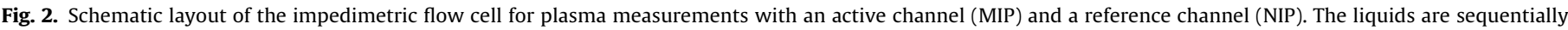
administered by a syringe-driven pump system. The temperature of the liquid is actively regulated for autonomous operation of the setup.

treatment guarantees the stability of serotonin [12]. After collection, the tubes were kept at $0{ }^{\circ} \mathrm{C}$ and subsequently centrifuged with $2000 \times \mathrm{g}$, corresponding to $19,620 \mathrm{~m} / \mathrm{s}^{2}$, for $10 \mathrm{~min}$ at $4{ }^{\circ} \mathrm{C}$ in order to obtain blood plasma. The as-prepared plasma samples were stored in Eppendorf tubes at $-80^{\circ} \mathrm{C}$ until analysis. For analysis, each plasma sample was divided into six different aliquots. One part was kept as a reference and remained unaltered, while the other parts were spiked with 50,100,150, 200 and $250 \mathrm{nM}$ serotonin. During handling, the samples were kept at $4{ }^{\circ} \mathrm{C}$ in to minimize enzymatic oxidation prior to analysis. Shortly before measuring, the samples were warmed up to $37^{\circ} \mathrm{C}$.

Reference HPLC measurements were conducted on the plasma samples of person B and C. These measurements were performed according to the protocol described by Danaceau et al. [12] in order to verify the native concentrations that were obtained by impedimetric read-out. For person B, the serotonin level according to HPLC was $11.2 \mathrm{nM}$ and for person $C$ the corresponding value was $8.9 \mathrm{nM}$.

\subsection{Impedance-spectroscopy platforms}

Two different impedimetric setups were used. For proof-ofprinciple experiments concerning the electronic recognition of serotonin in PBS buffer, we used an open impedimetric sensor cell, which was previously described for histamine detection [30]. The electrode couples (one couple for the MIP- and one for the NIP channel) have a planar geometry while the MIP/NIP powders are loaded onto the electrodes by using PPV as adhesive polymer in combination with stamping and a thermal treatment. For details of this immobilization procedure, we refer to [30,31]. The advantage of this open 'addition' cell is the large liquid volume, allowing to measure concentration series rapidly, without changing the liquid, by spiking up to higher and higher concentrations. The impedance signals were measured with an Iviumstat electrochemical analyzer from Ivium Technologies B.V. (Eindhoven, The Netherlands). Limitations of the open-cell concept with in-plane electrodes are the contact with ambient oxygen and impedimetric modeling requires assumptions concerning the current distribution.

In order to perform serotonin detection in blood plasma, a refined sensor-cell concept was developed shown in Fig. 2. This flow-through cell, made of Perspex, has an internal liquid volume of $110 \mu \mathrm{l}$. Patient's samples can be safely administered and oxidization of serotonin by ambient air is suppressed to the widest extend. The cell features also an integrated heating element and thermocouple, enabling stand-alone operation without need for temperature regulation in a furnace. During all measurements, the temperature was stabilized to $37 \pm 0.02{ }^{\circ} \mathrm{C}$ using a homemade PID controller. When dealing with a complex matrix such as blood derivatives, the non-specific responses from competing molecules can be stronger than the signals from specific recognition. To identify the specific signal nevertheless unambiguously, the MIP-functionalized electrode and the NIP-coated electrode (reference channel) were installed symmetrically with respect to a gold wire serving as a common counter electrode. This 'perpendicular' electrode configuration imposes also a well-defined current-flow direction through the MIP- or NIP-coated interface, thus facilitating the equivalent-circuit modeling. The electrodes here were made from $1 \mathrm{~cm} \times 1 \mathrm{~cm}$ aluminum substrates, coated again with the adhesive PPV polymer and loaded with equal amounts of MIP- and NIP powder. The contact area of each electrode with the liquid $\left(28 \mathrm{~mm}^{2}\right)$ was defined by $\mathrm{O}$-rings and the distance to the gold counter electrode was $1.7 \mathrm{~mm}$. Offset voltages between the gold electrode and the working electrodes could not be detected. The impedance signals were measured with a home-made, portable system operating in a frequency range of $100 \mathrm{~Hz}$ to $100 \mathrm{kHz}$ with 10 frequencies per decade and a scanning speed of $5.7 \mathrm{~s}$ per sweep $[34,35]$. The amplitude of the AC voltage was fixed to $10 \mathrm{mV}$.

\section{Results and discussion}

\subsection{Optical batch rebinding experiments}

For all four monomer blends mentioned under Section 2.2, the performance of the obtained MIPs was analyzed by batch rebinding experiments. For these experiments, $50 \mathrm{mg}$ of MIP- or NIP powder were added to $5 \mathrm{ml}$ of aqueous serotonin concentrations in the range between 0.1 and $1 \mathrm{mM}$. The resulting suspensions were shaken for $2 \mathrm{~h}$ on a rocking table at room temperature. After filtration, the free concentration $\left(C_{\mathrm{f}}\right)$ of serotonin was measured by UV-vis spectroscopy. Hereafter, the amount of bound serotonin per gram MIP or NIP was calculated $\left(S_{\mathrm{b}}\right)$ and the binding isotherms and the corresponding affinity distributions were determined. Fig. 3(a) shows the binding isotherm of the optimal MIP with the $1: 3$ ratio between MAA and AM. The solid line is based on a two-parameter fit of the type $S_{\mathrm{b}}=A C_{\mathrm{f}}^{\nu}$. This mathematical expression corresponds to a Freundlich isotherm, which is expected in a MIP system with a heterogeneous distribution of binding sites and affinity constants [36] There is a clear difference between the MIP- and the NIP isotherm and, when looking at a concentration $C_{\mathrm{f}}=0.3 \mathrm{mM}$, the imprint 

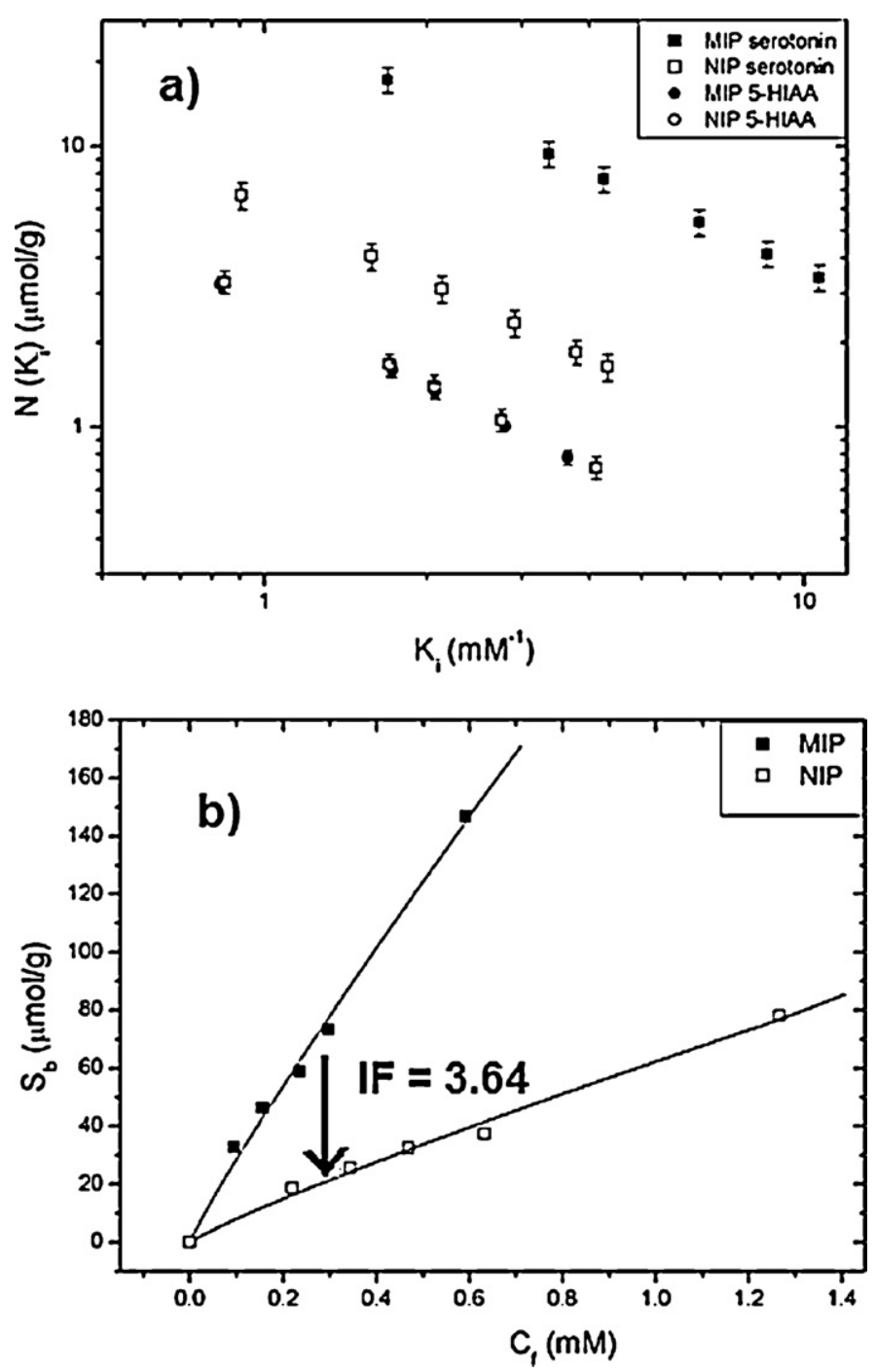

Fig. 3. The upper panel (a) shows the binding isotherms of MIPs (solid squares) and the corresponding NIPs (open squares) upon exposure to serotonin. Solid lines are based on the allometric fit described in the text; the imprinting factor for optimized monomer blend is close to 4. (b) Shows the corresponding affinity distributions for MIPs and NIPs (solid and open squares) according to the Freundlich model. The affinity for the competitor molecule 5-HIAA (solid and open circles) is negligible both for the MIP and the NIP.

factor IF is 3.6 while other concentrations give similar IF values. This confirms recent findings by Khurshid et al., who reported that a blend of charged and neutral monomers, as also used in our study, is crucial for obtaining serotonin MIPs with good affinity properties [28]. In contrast to this, MIPs for the recognition of nicotine or histamine can be synthesized by using MAA as a single, neutral monomer $[29,30]$.

The affinity distribution, as derived from the binding isotherms, is shown in Fig. 3(b). By using the Freundlich model, the total number of binding sites, $N_{\text {tot }}$, within the range of the affinity constants $K_{\mathrm{i}}=1-15 \mathrm{mM}^{-1}$ can be calculated for the MIP and NIP. In this region the number of binding sites for the MIP is $26 . \pm 2.1 \mu \mathrm{mol} / \mathrm{g}$ and for the corresponding NIP $5.6 \pm 0.4 \mu \mathrm{mol} / \mathrm{g}$. This indicates that, within the considered range, the MIP has 4.7 times the amount of binding sites as compared to the NIP. For comparison, a Langmuir interpretation (all binding sites assumed to be equivalent) also confirms the stronger affinity of the $\operatorname{MIP}\left(K=3.6 \pm 0.1 \mathrm{mM}^{-1}\right)$ as compared to the NIP $\left(1.2 \pm 0.03 \mathrm{mM}^{-1}\right)$. However, the Scatchard plot clearly indicated heterogeneous behavior and therefore the Langmuir model is not applicable to this system. Another alternative is a combination of Freundlich and Langmuir behavior, which resulted also in a fit with a high linearity coefficient $\left(R^{2}=0.99\right)$ [37]. We will show here only the results of the Freundlich model as this gave an equally good fit $\left(R^{2}=0.99\right)$ as the Freundlich-Langmuir model using one parameter less.

In order to test the selectivity of the molecular recognition, the MIPs and NIPs were also exposed to the natural metabolite of serotonin (5-HIAA) and to its chemical oxidation product (Fig. 1(b) and (c)). To obtain the oxidized version, serotonin solutions $(0.1-1 \mathrm{mM})$ were intentionally left in contact with air for one week, resulting in a brownish discoloration even for the lowest concentration. The addition of MIP- or NIP powder could not remove this discoloration and therefore UV-vis measurements were not feasible. However, this also suggests that the binding of the oxidized molecules by serotonin MIPs is negligible, which will be confirmed by the impedance data presented in Section 3.2. In case of 5-HIAA, we could extract UV-vis data and in the affinity-constant region of $1-15 \mathrm{mM}^{-1}$ the number of binding sites for the MIP is $2.6 \pm 0.1 \mu \mathrm{mol} / \mathrm{g}$ and for the NIP $2.6 \pm 0.2 \mu \mathrm{mol} / \mathrm{g}$, see Fig. 3(b). This proofs that the limited binding of the metabolite must be entirely due to non-specific adsorption, which is even less than the nonspecific adsorption of serotonin to the NIPs. A reason may be the acidic character of the $\mathrm{COOH}$ groups present in the metabolite.

\subsection{Impedance spectroscopy in PBS buffer with open sensor cell}

MIP- and NIP powders were immobilized on the planar electrode structures as mentioned in Section 2.4. By optical microscopy in combination with image processing by Image $\mathrm{J}$ software we verified that in case of the MIP a surface coverage of $28 \pm 1.2 \%$ was obtained, while it was $27 \pm 1.3 \%$ for the NIP-loaded electrode. This shows that an almost identical surface coverage can be readily achieved while also the particle-size distribution of MIP and NIP is similar. Furthermore, the surface coverage was validated by the change in fluorescence intensity with a Zeiss LSM 510 Meta Axiovert $200 \mathrm{M}$ laser scanning confocal fluorescence microscopy using $514 \mathrm{~nm}$ argon-ion laser excitation. The MDMO-PPV shows strong fluorescence [33], while for the MIP and NIP this is not the case and therefore a sharp contrast can be seen between the particles and the background. Subsequently, the fluorescence images processed by Zeiss LSM Image Browser determined a $27 \pm 3.0 \%$ coverage for the MIP and a $25 \pm 2.5 \%$ coverage for the NIP. From SEM-analysis, the thickness of the layers was determined to be $4.0 \pm 0.5 \mu \mathrm{m}$. Therefore, the precondition for differential measurements, having identical particle loadings, is fulfilled. Next, the sensor cell was filled with PBS buffer in order to simulate the $\mathrm{pH}$ and ionic strength of human blood. Also, the physiological temperature conditions of $37^{\circ} \mathrm{C}$ were mimicked. After stabilizing for $25 \mathrm{~min}$, increasing concentrations of serotonin were added in a stepwise manner, resulting in a concentration series from 0 to $64.5 \mathrm{nM}$ with an increment of $10.75 \mathrm{nM}$ per step. The corresponding dose-response curve at a frequency of $213 \mathrm{~Hz}$ is given in Fig. 4. All data were actually measured in the entire available frequency range. The $213 \mathrm{~Hz}$ data presented here were selected for the very good signal-to-noise ratio while this frequency is low enough to probe capacitive interface effects.

The impedance data for each concentration are normalized with respect to a starting value of $100 \%$ for pure, serotonin-free PBS solution. The highest serotonin concentration $(64.5 \mathrm{nM})$ resulted in an impedance increase of $8.4 \pm 0.4 \%$ while physiologically relevant concentrations in the order of $10 \mathrm{nM}$, see, e.g. Ref. [11], also result in an easily measurable increase of $2.5 \% \pm 0.2 \%$. The error at this concentration is used to estimate the detection limit, which is commonly defined as the concentration where the signal amplitude equals three times the standard deviation. In the low-concentration regime $(0-30 \mathrm{nM})$, the dose-response results can be represented 


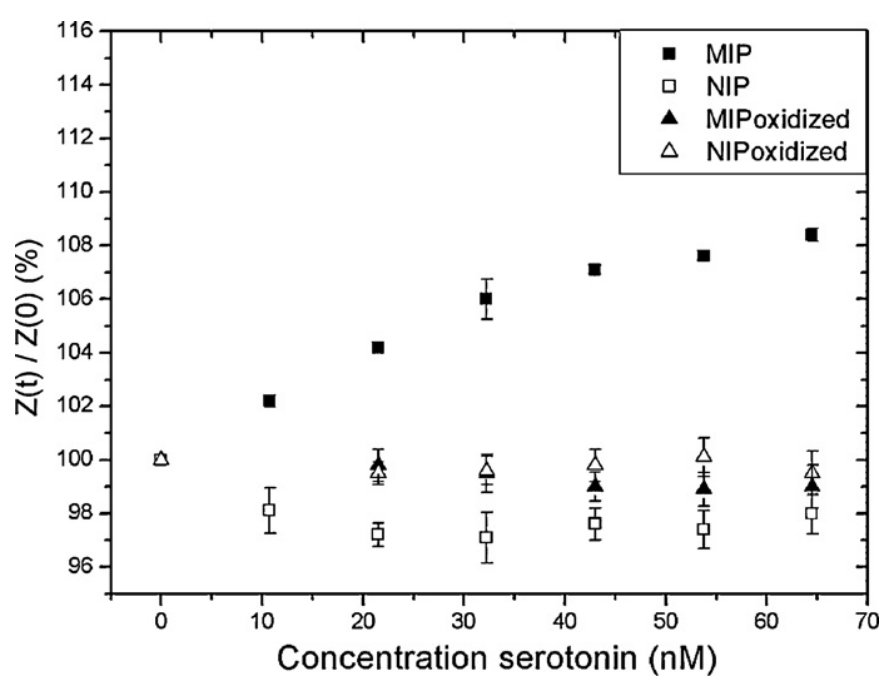

Fig. 4. Dose-response curves for MIP and NIP exposed to increasing concentrations of serotonin and the oxidized form of serotonin in PBS buffer. For exposure to serotonin, data points are shown as solid squares (MIP) and open squares (NIP). For exposure to the oxidized form of serotonin the data are given as solid triangles (MIP) and open triangles (NIP). The sensor response to the oxidized version of serotonin is negligible. The error bars for the MIP are smaller than the symbol size.

well with a linear fit $\left(R^{2}=0.99\right)$. With this obtained fit, the limit of detection was calculated to be $3.2 \mathrm{nM}$. As this is below the physiologically relevant concentrations, we can use the sensor for medical applications.

For concentrations higher than $40 \mathrm{nM}$, the dose-response curve shows a trend toward saturation that can be attributed to the increasing occupation of binding sites. The NIP-based electrode gave no clear impedance change beyond experimental uncertainties. During these measurements we also took into account that the molecular recognition of the target molecules by MIPs is not instantaneous: after each increase of the serotonin concentration, the sensor cell was allowed to stabilize for $10 \mathrm{~min}$ and then the impedance was measured for six times with an intermediate waiting time of $3 \mathrm{~min}$. This 'moving average filter' reduces data scattering to a minimum and the total measuring time for a specific concentration is still not more than $30 \mathrm{~min}$.

To test the selectivity of the sensor, measurements were also performed with PBS solutions containing the oxidized version of serotonin with the same sequence of concentration steps. The chemical structure is similar, however due to oxidation less hydrogen bonds can be formed and therefore binding to the nanocavities is hampered. As shown in Fig. 4, neither the MIP- nor the NIP-coated electrode shows a measurable response to the oxidized molecules. Together with the optical batch-rebinding data, proving that the MIPs do not bind the metabolite 5-HIAA, we can indeed conclude that the recognition of serotonin is selective. Therefore, the sensor is insensitive toward the most important competitor molecules, which are naturally present in patients' samples.

\subsection{Serotonin detection in human blood plasma with a flow-through impedimetric sensor cell}

For these measurements, the cell was first filled with unaltered (non-spiked) plasma of person A, which was prepared according to Section 2.3. The sensor cell was allowed to stabilize $90 \mathrm{~min}$ and the impedance spectra of the MIP- and NIP channel were measured, again with the moving average filter. The resulting impedance values at $213 \mathrm{~Hz}$ were used as a nominal sensor baseline, irrespective of the $a$ priori unknown concentration $c_{0}$ of native serotonin present in the plasma sample. Note that even the lowest of the
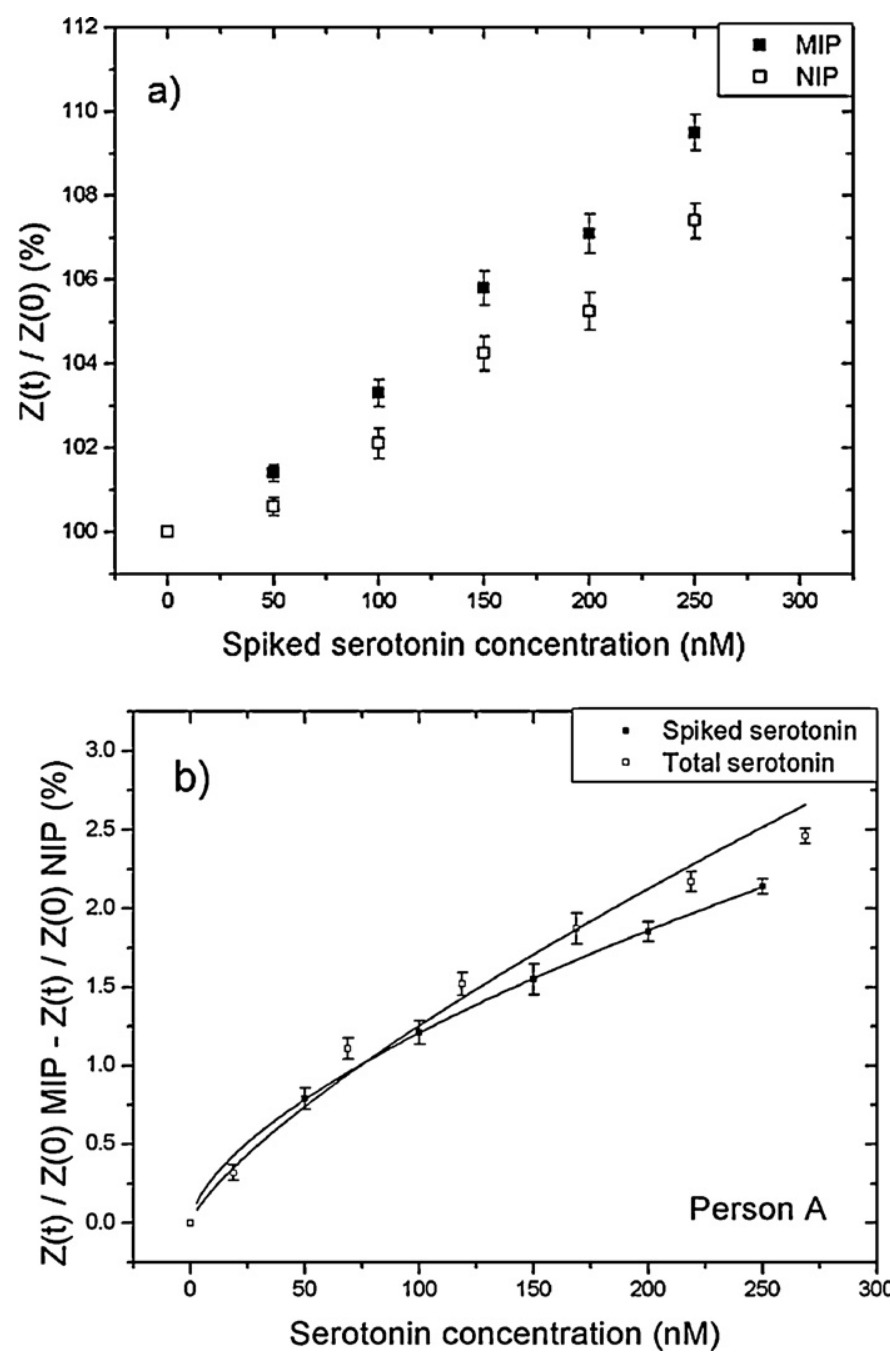

Fig. 5. The upper panel (a) shows the normalized impedance response of the MIP and the NIP-based sensor electrode to plasma samples of person A spiked with serotonin. The lower panel (b) shows the differential signal as a function of the spiked concentration by solid squares. The open squares represent the dose-response data obtained after correction for the native serotonin concentration $c_{0}(18.8 \mathrm{nM})$ present in the person's plasma. The solid lines are allometric fits as described in the text.

spiked concentrations, $50 \mathrm{nM}$, is at least 2.5 times higher than the typical physiological concentrations of 5-20 nM. Therefore, the intrinsic $c_{0}$ value is expected to be a minor correction to the final dose-response curve. Next, the spiked plasma samples were introduced one by one via the pumping system. After waiting each time for $20 \mathrm{~min}$, the impedance spectra of the MIP- and NIP channel were determined before switching to the next-higher concentration. The impedance values for the MIP- and the NIP electrode, normalized to non-spiked plasma, are given in Fig. 5(a).

As compared to the studies with PBS electrolyte, the impedance change of the MIP electrode upon exposure to a given serotonin concentration is less pronounced. This stems possibly from the non-specific adsorption of plasma proteins on the electrode, which results in a partial blocking of the MIP nanocavities. The response of the NIP electrode to serotonin seems stronger as compared to the data in PBS (see Fig. 4), but we are dealing now with considerably higher concentrations. In any case, there is a clearly visible difference between the impedance change of the MIP- and NIP channel, which is getting larger when increasing the spiked concentration. The differential signal $Z / Z(0)\{\mathrm{MIP}\}-Z / Z(0)\{\mathrm{NIP}\}$ is shown in Fig. 5(b). This differential dose-response curve can excellently be described with an allometric fit according to $y=a c^{b}$. Hereby, $y$ is the 
differential impedance change in percent while $c$ is the concentration in nanomolar units. The fit parameters $a$ and $b$ have the value $a=0.07 \% \pm 0.002 \%$ and $b=0.62 \pm 0.005$, the coefficient of determination $R^{2}$ is 0.99 . Although we are not discussing a binding isotherm in the strict sense, the similarity with the Freundlich isotherm (see Section 3.1 above) is obvious.

In order to estimate also the concentration $c_{0}$ of native serotonin, we determined in a second step the sensor baseline more precisely by filling the sensor cell with serotonin-free plasma. For this, $10 \mathrm{mg}$ of MIP powder was added to $3 \mathrm{ml}$ of unaltered plasma and this mixture was shaken for $30 \mathrm{~min}$ on a rocking table, allowing the MIPs to absorb the native serotonin of person A. According to the binding isotherms, we assumed the serotonin to be extracted completely from the liquid and subsequently the particles were removed by filtration. Furthermore, the cell was equipped with freshly prepared MIP- and NIP electrodes and filled subsequently with the serotoninfree and with the unaltered plasma samples. After stabilization each time at $37^{\circ} \mathrm{C}$ for $30 \mathrm{~min}$, the impedance values were determined. The unaltered sample gave a differential-impedance increase of $0.3 \pm 0.03 \%$ as compared to the starting value with the serotoninfree plasma filling. In the sense of the differential dose-response curve, this corresponds to a native concentration of $c_{0}=19 \pm 1.2 \mathrm{nM}$, well within the physiologically expected range [10]. Based on these offset values, the dose-response curve of Fig. 5(b) was rescaled by adding $c_{0}$ to all spiked concentrations and by shifting the differential impedance increases with $+0.32 \%$. The resulting data points can again be described with the fit $y=a^{\prime} c^{b^{\prime}}$ with the parameters $a^{\prime}=0.06 \% \pm 0.02 \%$ and $b^{\prime}=0.7 \pm 0.05 \%$. The $R^{2}$ value is 0.98 . The detection limit in plasma was determined according to the procedure described in Section 3.2 for the buffer measurements. The standard deviation was taken at the lowest spiked concentration $(50 \mathrm{nM})$. The data in the low-concentration regime was fitted linearly $\left(R^{2}=0.94\right)$, which resulted in a detection limit of $4.3 \mathrm{nM}$. This value is slightly higher compared to what was obtained in buffer measurements ( $3.2 \mathrm{nM})$, but is still below the physiologically relevant concentration range.

To validate the results, two additional samples of person $B$ and $C$ were measured by our technique and compared with corresponding HPLC measurements performed according to the method established by Danaceau et al. [12]. First, a calibration curve was constructed for person $\mathrm{B}$ by extracting the native concentration from the plasma and then measuring the impedance response to the spiked concentrations 15, 25, 100, 150 and $200 \mathrm{nM}$ (Fig. 6). The dose response curve can be described with the fit $y=a^{\prime} c^{b^{\prime}}$ with the parameters $a^{\prime}=0.03 \% \pm 0.007 \%$ and $b^{\prime}=0.8 \pm 0.06 \%$ and has an $R^{2}$ value of 0.97 .

Subsequently, a second measurement was performed with an unaltered sample which showed an impedance response of $+0.27 \%$. With the obtained calibration curve, the native serotonin concentration of patient $B$ was determined to be $13.3 \pm 1.3 \mathrm{nM}$. The HPLC reference test indicated a concentration of $11.2 \mathrm{nM} \pm 1.0 \mathrm{nM}$, as mentioned in Section 2.3. The same procedure was repeated for person $\mathrm{C}$. When using our technique a concentration of $9.5 \pm 1.0 \mathrm{nM}$ was obtained, while the HPLC measured a concentration of $8.9 \mathrm{nM} \pm 0.5 \mathrm{nM}$. Hereby, it is demonstrated that our sensor provides indeed reliable data in the diagnostically relevant concentration regime.

\subsection{Response modeling of plasma measurements with equivalent circuits}

As a final step, the origin of the impedance increase upon serotonin binding was further analyzed for the plasma samples of patient A. Fig. 7(a) shows the Bode plot in the frequency regime from $100 \mathrm{~Hz}$ to $100 \mathrm{kHz}$ for the MIP electrode when the cell was filled with unaltered plasma. The solid line was calculated with

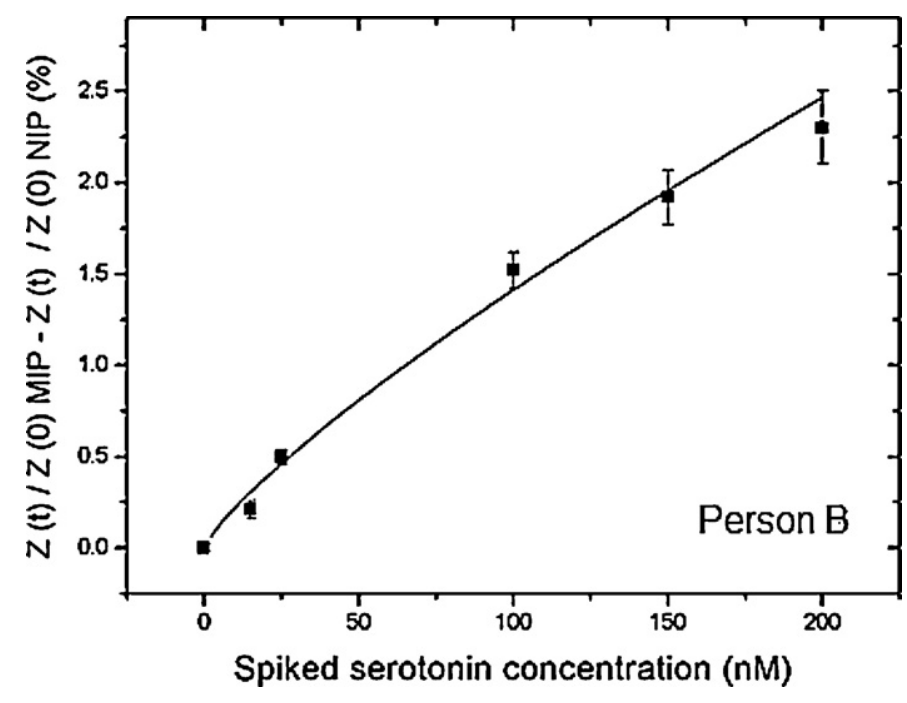

Fig. 6. The normalized impedance response obtained for extracted plasma sample of person B spiked with $15,25,100,150$ and $200 \mathrm{nM}$. The derived native concentration for derived from this calibration curve is $13.3 \pm 1.3 \mathrm{nM}$ which is in agreement with the HPLC result of $11.2 \pm 1.0 \mathrm{nM}$.

the equivalent circuit given in Fig. 7(b): $R_{\mathrm{f}}$ refers to the resistance of the plasma and the counter electrode, elements that are not expected to change upon spiking with higher serotonin concentrations. The adhesive PPV layer and aluminum substrate are in series with $R_{\mathrm{f}}$ and can be represented by an element with a resistor $R_{\mathrm{PPV}}$ and a constant-phase element (leaking capacitor) CPEPPV in parallel. A leaking capacitor was chosen to take into account roughness and inhomogeneity on the polymeric electrode surface. Finally, also the MIP layer, covering roughly $30 \%$ of the electrode's surface, was described as a CPE $\mathrm{MIP}$ in series. Here, a leaking capacitor is expected
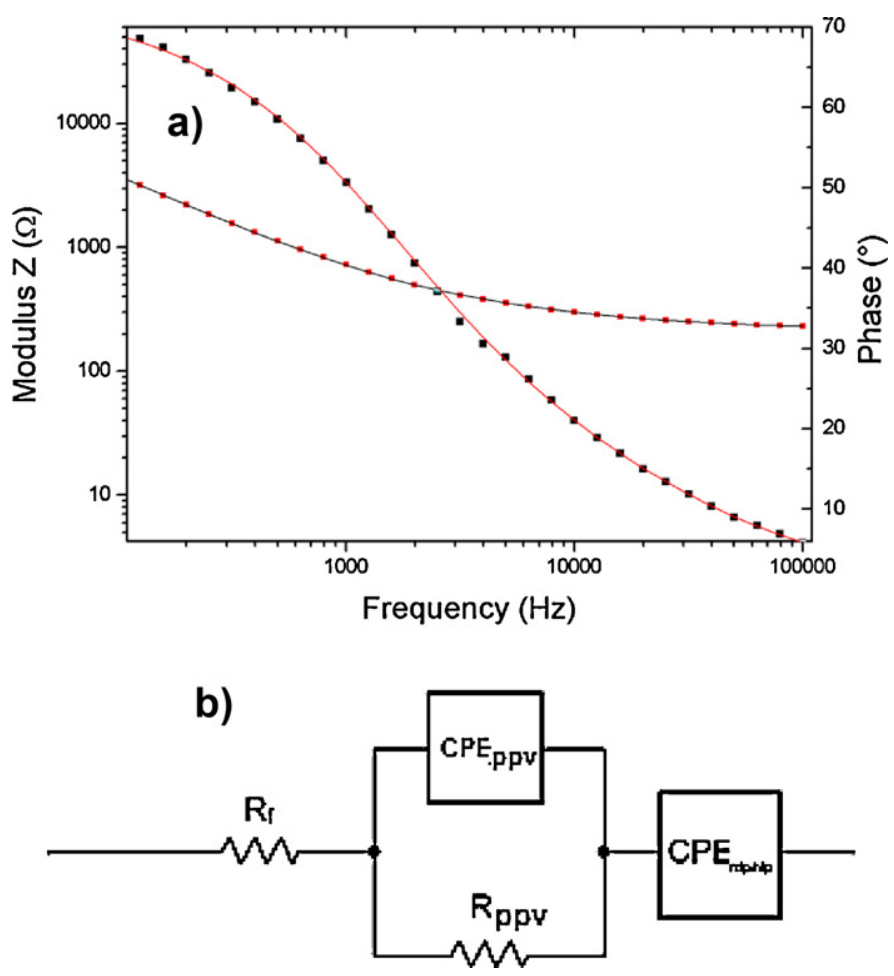

Fig. 7. The upper panel (a) shows the Bode plot of the MIP electrode when exposed to blood plasma with the native serotonin concentration $c_{0}$. The solid lines are calculated by using the equivalent circuit shown in (b) in combination with the fit parameters given in Table 1. 
Table 1

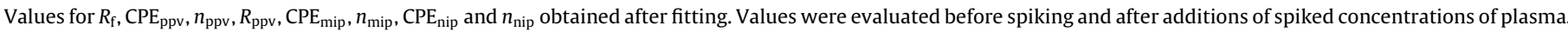

\begin{tabular}{|c|c|c|c|c|c|c|c|c|c|c|c|c|c|c|}
\hline \multicolumn{8}{|l|}{ MIP } & \multicolumn{7}{|l|}{ NIP } \\
\hline Spiked (nM) & $R_{\mathrm{f}}(\Omega)$ & $\mathrm{CPE}_{\mathrm{ppv}}\left(\mu \mathrm{S} \times \mathrm{s}^{n}\right)$ & $n_{\mathrm{ppv}}$ & $R_{\mathrm{ppv}}(\Omega)$ & $\mathrm{CPE}_{\text {mip }}\left(\mu \mathrm{S} \times \mathrm{s}^{n}\right)$ & $n_{\text {mip }}$ & $\chi^{2}\left(10^{-4}\right)$ & $R_{\mathrm{f}}(\Omega)$ & $\mathrm{CPE}_{\mathrm{ppv}}\left(\mu \mathrm{S} \times \mathrm{s}^{n}\right)$ & $n_{\mathrm{ppv}}$ & $R_{\mathrm{ppv}}(\Omega)$ & $\mathrm{CPE}_{\text {nip }}\left(\mu \mathrm{S} \times \mathrm{S}^{n}\right)$ & $n_{\text {nip }}$ & $\chi^{2}\left(10^{-4}\right)$ \\
\hline 0 & 212 & 1.48 & 0.79 & 118 & 13 & 0.62 & 1.52 & 228 & 1.71 & 0.80 & 132 & 54 & 0.46 & 0.82 \\
\hline 50 & 218 & 1.41 & 0.82 & 88 & 7.0 & 0.69 & 1.06 & 232 & 1.56 & 0.81 & 131 & 53 & 0.46 & 0.30 \\
\hline 100 & 220 & 1.36 & 0.83 & 80 & 5.3 & 0.72 & 1.49 & 232 & 1.50 & 0.83 & 125 & 52 & 0.46 & 0.36 \\
\hline 150 & 220 & 1.32 & 0.84 & 75 & 3.9 & 0.75 & 2.97 & 233 & 1.46 & 0.84 & 109 & 45 & 0.48 & 0.40 \\
\hline 200 & 222 & 1.30 & 0.85 & 71 & 2.8 & 0.79 & 4.93 & 233 & 1.43 & 0.85 & 117 & 50 & 0.47 & 0.44 \\
\hline 250 & 228 & 1.27 & 0.86 & 72 & 3.4 & 0.77 & 4.93 & 234 & 1.44 & 0.85 & 99 & 36 & 0.51 & 0.48 \\
\hline
\end{tabular}

due to the intrinsic porosity of the MIP material. The NIP channel was described by a corresponding $\mathrm{CPE}_{\mathrm{NIP}}$.

The parameters derived from the fit by using online available ZSimpWin software are summarized in Table 1 . The high quality of the fit is illustrated by a $\chi^{2}$ value around $1 \times 10^{-4}$. Other equivalent circuit models based on the same number of elements had a $\chi^{2}$ of $10^{-3}$ and were clearly less accurate. A more refined modeling of the MIP layer, e.g. in terms of a resistor and a CPE in parallel, gave also $\chi^{2}$ in the order of $10^{-4}$, meaning that the proposed circuit is indeed an excellent description of the system with a minimum number of elements. The same circuit also describes the behavior of the NIP electrode with a similarly low $\chi^{2}$ and can still be applied after exposing the MIP- and NIP electrodes to the spiked serotonin concentrations. All extracted data are summarized in Table 1.

When comparing the MIP- and the NIP channel for the different spiking concentrations, we note first that the values of the liquid resistance $R_{\mathrm{f}}$, the amplitude of the constant phase element CPE $E_{P P V}$ and its exponent $n_{\mathrm{PPV}}$, and the resistance $R_{\mathrm{PPV}}$ are widely constant. The strongest variation is seen in $R_{\mathrm{PPV}}$ where absolute values can differ by a maximum of $50 \%$ when comparing the value for MIP and NIP at a given spiking concentration. The exponent $n_{\text {PPV }}$ stays always in the range of $0.79-0.86$, which is close to the behavior of an ideal capacitor $(n=1)$. When comparing the CPE behavior of the MIP- and NIP element, the differences become immediately much more striking: the NIP electrode has no serotonin-shape nanocavities and the absolute CPE amplitude is therefore insensitive to the spiked concentration while the exponent $\left(n_{\text {NIP }}=0.46-0.51\right)$ resembles closely a Warburg behavior. The MIP electrode has a much lower CPE amplitude for the non-spiked sample as compared to the NIP electrode (25\%) and this decreases systematically to $10 \%$ of the NIPs value when the MIP electrode was exposed to the highest serotonin concentration. At the same time, the exponent $n_{\mathrm{MIP}}$ shifts up from Warburg-like to capacitor-like behavior. Fig. 8 shows the concentration-dependent CPE amplitude of the MIP- and NIP electrode normalized to the initial value obtained in non-spiked plasma.

The current data do not yet allow interpreting the resistiveand capacitive aspects of the CPE-behavior on morphological or even molecular grounds, but a coarse-grain physical interpretation may be as follows: the CPE behavior of the MIP electrode is always more on the capacitive than on the resistive side. The absolute capacity value (related to the amplitude of the CPE element) decreases for increasing serotonin concentration, which means that the serotonin-sized cavities in the MIP polymer are more and more filled by serotonin- rather than by water molecules. As a result, the effective contact area between the MIP polymer and the electrolyte (here: plasma) decreases while water, with its high dielectric constant of $\varepsilon=81$, is replaced by organic molecules with $\varepsilon$ in the range of 5-10 [38]. Both aspects, the shrinking of the contact area and the decrease of the dielectric constant at the solid-liquid interface, work into the direction of lowering the electronic interface capacity, which corroborates the data shown in Fig. 8.

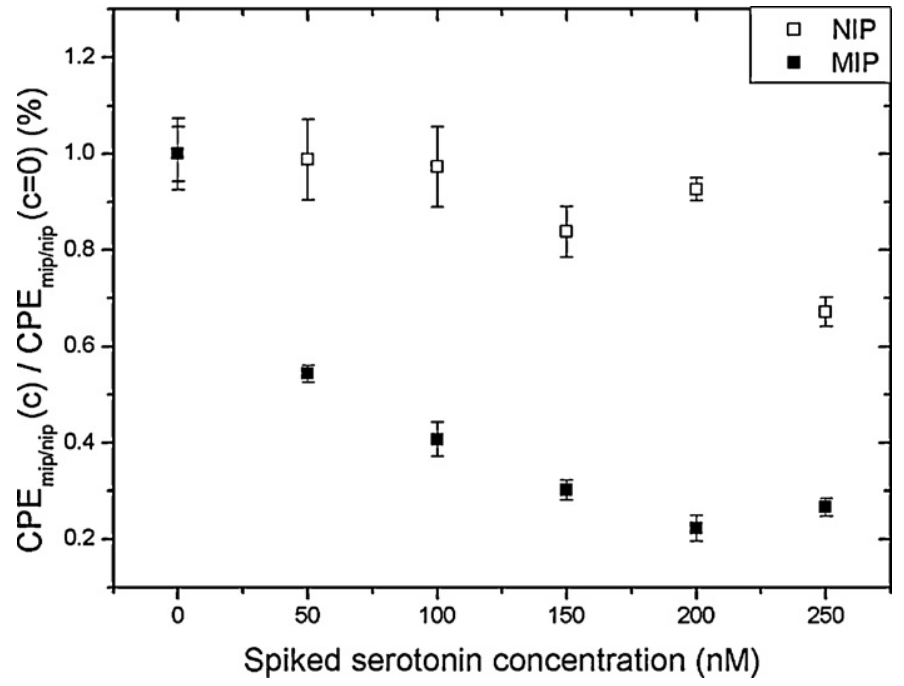

Fig. 8. Concentration dependence of the amplitude of the constant-phase element representing the MIP-type receptors (solid squares) and the NIP reference material (open squares). The change of the CPE behavior is responsible for the increasing sensor impedance with increasing serotonin level.

\section{Conclusions}

In this work, we have shown that a biomimetic serotonin sensor, based on impedance spectroscopy in combination with MIP-based synthetic receptors, has the potential to become a fast and lowcost alternative to chromatographic techniques. At the receptor side, we have shown that a blend of two functional monomers, methacrylic acid and acrylamide, is essential to achieve selective molecular recognition and the best properties were obtained with a MAA-AM ratio of 1:3. Furthermore, we have demonstrated that the sensor response is specific and selective with respect to its competitors, metabolite 5-HIAA and the oxidized version of serotonin. At the sensor side, we developed a symmetrical flow-through cell hosting an active channel (MIP) and a reference channel NIP in order to correct for unwanted side effects such as protein absorption, which naturally occur in a biological matrix. The MIPs as such can also be used to extract the native serotonin from a patient's sample in order to obtain a well-defined sensor baseline in the limit of vanishing serotonin concentration. The detection limit is below the physiologically relevant range and the sensor can operate in nondiluted blood plasma provided that standard precautions are taken to counteract oxidization and enzymatic conversion of serotonin. Furthermore, the determined concentrations show comparatively small uncertainties and are in perfect agreement with HPLC reference tests, as was illustrated for two different persons. This cell allows for the safe and temperature-controlled handling of plasma samples. Moreover, in combination with a compact impedance analyzer, the sensor setup can be operated as a 'stand-alone device', 
making it well suited for point-of-care applications like, e.g. in a hospital environment.

\section{Acknowledgments}

This work is supported by the School for Life-Sciences of the transnational University Limburg, the Life-Science Initiative of the Province of Limburg, the Special Research Funds BOF of Hasselt University and an IMEC-PhD fellowship. Technical support by $\mathrm{H}$. Penxten, J. Soogen, J. Baccus, H. Strauven, Dr. J. d'Haen and Prof. M. Ameloot is gratefully acknowledged.

\section{References}

[1] V. Esparmer, G. Boretti, Identification and characterization by paper chromatography of enteramine, octopamine, tyramine, histamine and allied substances in extracts of posterior salivary glands of octopoda and in other tissue abstracts of vertebrates and invertebrates, Archives of International Pharmacodynamics 88 (1951) 296-332.

[2] I.P. Kema, E.G.E. De Vries, F. Muskiet, Clinical chemistry of serotonin and metabolites, Journal of Chromatography B: Analytical Technologies in the Biomedical and Life Sciences 747 (2000) 33-48.

[3] J.E. Blundell, Serotonin and the biology of feeding, American Journal of Clinical Nutrition 55 (1992) 155S-159S.

[4] J.R. Seibold, Serotonin and the cardiovascular system, Raven Press, New York, 1985.

[5] D.G. Grahame-Smith, The carcinoid syndrome, American Journal of Cardiology 21 (1968) 376-385.

[6] A.N.M. Wymenga, T.A. Van der Graaf, I.P. Kema, E.G.E. De Vries, N.H. Mulder, Effects of peripheral stem-cell or bone-marrow reinfusion on peripheral serotonin metabolism, Lancet 353 (1999) 293-294.

[7] M. Costedio, N. Hyman, G. Mawe, Serotonin and its role in colonic function and in gastrointestinal disorders, Diseases of the Colon and Rectum 50 (2005) 376-388.

[8] M.D. Crowell, Role of serotonin in the pathophysiology of the irritable bowel syndrome, British Journal of Pharmacology 141 (2009) 1285-1293.

[9] D. Keszthelyi, F.J. Troost, A.A.M. Masclee, Understanding the role of tryptophan and serotonin metabolism in gastrointestinal function, Neurogastroenterol ogy and Motility: the official Journal of the European Gastrointestinal Motility Society 21 (2009) 1239-1249.

[10] G.M. Anderson, J.M. Stevenson, D.J. Cohen, Steady-state model for plasma free and platelet serotonin in man, Life Sciences 41 (1987) 1777-1785.

[11] G.M. Anderson, Signal-to-noise optimization of HPLC-fluorometric systems and their application to the analysis of indoles, Advances in Experimental Medicine and Biology 294 (1991) 51-61.

[12] J.P. Danaceau, G.M. Anderson, W.M. McMahon, D.J. Crouch, A liquid chromatographic-tandem mass spectrometric method for the analysis of serotonin and related indoles in human whole blood, Journal of Analytical Toxicology 27 (2003) 440-444.

[13] D. Tonelli, E. Gattavecchia, M. Gandolfi, Thin-layer chromatographic determination of indolic tryptophan metabolites in human urine using Sep-Pak $\mathrm{C}_{18}$ extraction, Journal of Chromatography B: Analytical Technologies in the Biomedical and Life Sciences 231 (1982) 283-289.

[14] B.A. Patel, M. Arundell, K.H. Parker, M.S. Yeoman, D. O'Hare, Simple and rapid determination of serotonin and catecholamines in biological tissue using highperformance liquid chromatography with electrochemical detection, Journal of Chromatography B: Analytical Technologies in the Biomedical and Life Sciences 818 (2005) 269-276.

[15] B.V. Sarada, Tata N. Rao, D.A. Tryk, A. Fujishima, Electrochemical oxidation of histamine and serotonin at highly boron-doped diamond electrodes, Analytical Chemistry 72 (2000) 1632-1638

[16] K. Wu, J. Fei, S. Hu, Simultaneous determination of dopamine and serotonin on a glassy carbon electrode coated with a film of carbon nanotubes, Analytical Biochemistry 318 (2003) 100-106.

[17] B.E. Kumara Swamy, B.J. Venton, Carbon nanotube-modified microelectrodes for simultaneous detection of dopamine and serotonin in vivo, Analyst 132 (2007) 876-884

[18] G. Wulff, The role of binding site interactions in the molecular imprinting of polymers, Trends in Biotechnology 11 (1993) 85-87.

[19] R. Arshady, K. Mosbach, Synthesis of substrate-selective polymer by host-guest polymerization, Chemical Physics 182 (1981) 687-692.

[20] K. Mosbach, Molecular imprinting, Trends in Biochemical Sciences 19 (1994) 9-14

[21] P.K. Owens, L. Karlsson, E.S.M. Lutz, L.I. Andersson, Molecular imprinting for bioand pharmaceutical analysis, TrAC Trends in Analytical Chemistry 18 (1999) 146-154.

[22] S.A. Piletsky, S. Alcock, A.P.F. Turner, Molecular imprinting: at the edge of the third millennium, Trends in Biotechnology 19 (2001) 9-12.

[23] B. Sellergren, C.J. Allender, Molecularly imprinted polymers: a bridge to advanced drug delivery, Advanced Drug Delivery Reviews 57 (2005) 1733-1741.
[24] D.A. Spivak, Optimization, evaluation, and characterization of molecularly imprinted polymers, Advanced Drug Delivery Reviews 57 (2005) 1779-1794.

[25] T. Kitade, K. Kitamura, T. Konishi, S. Takegami, T. Okuno, M. Ishikawa, M. Wakabayashi, K. Nishikawa, Y. Muramatsu, Potentiometric immunosensor using artificial antibody based on molecularly imprinted polymers, Analytical Chemistry 76 (2004) 6802-6807.

[26] B. Okutucu, A. Telefoncu, Optimization of serotonin imprinted polymers and recognition study from platelet rich plasma, Talanta 76 (2008) 1153-1158.

[27] B. Okutucu, A. Telefoncu, K. Haupt, Molecularly imprinted polymer for serotonin recognition, Haceteppe Journal of Biology and Chemistry 38 (2010) 79-84.

[28] S.S. Khurshid, C.E. Schmidt, N. Peppas, Optimization of molecularly imprinted polymers of serotonin for biomaterial applications, Journal of Biomaterials Science Polymer Edition 22 (2011) 343-362.

[29] R. Thoelen, R. Vansweevelt, J. Duchateau, F. Horemans, J. D‘Haen, L. Lutsen, D. Vanderzande, M. Ameloot, M. vandeVen, T.J. Cleij, P. Wagner P, A MIP-based impedimetric sensor for the detection of low-MW molecules, Biosensors and Bioelectronics 23 (2008) 913-918.

[30] E. Bongaers, J. Alenus, F. Horemans, A. Weustenraed, L. Lutsen, D. Vanderzande, T.J. Cleij, F.J. Troost, R.-J. Brummer, P. Wagner, A MIP-based biomimetic sensor for the impedimetric detection of histamine in different $\mathrm{pH}$ environments, Physica Status Solidi A 207 (2010) 837-843.

[31] F. Horemans, J. Alenus, E. Bongaers, A. Weustenraed, R. Thoelen, J. Duchateau, L. Lutsen, D. Vanderzande, P. Wagner, T.J. Cleij, MIP-based sensor platforms for the detection of histamine in the nano- and micromolar range in aqueous media, Sensors and Actuators, B: Chemical 148 (2010) 392-398.

[32] M.C. Blanco-López, M.J. Lobo-Castañón, A.J. Miranda-Ordieres, P. Tuñón-Blanco, Electrochemical sensors based on molecularly imprinted polymers, TrAC Trends in Analytical Chemistry 2 (2004) 36-48.

[33] F. Louwet, D. Vanderzande, J. Gelan, A general synthetic route to high molecular weight poly(p-xylylene)-derivatives: a new route to poly(p-phenylene vinylene), Synthetic Metals 69 (1995) 509-510.

[34] B. van Grinsven, T. Vandenryt, S. Duchateau, A. Gaulke, L. Grieten, R. Thoelen, S. Ingebrandt, W. De Ceuninck, P. Wagner, Customized impedance spectroscopy device as possible sensor platform for biosensor applications, Physica Status Solidi A 207 (2011) 919-923.

[35] B. van Grinsven, N. Vanden Bon, L. Grieten, M. Murib, S. Janssens, K. Haenen, E. Schneider, S. Janssens, K. Haenen, E. Schneider, S. Ingebrandt, M.J. Schöning, V. Vermeeren, M. Ameloot, L. Michiels, R. Thoelen, W. De Ceuninck, P. Wagner, Rapid assessment of the stability of DNA duplexes by impedimetric real-time monitoring of chemically induced denaturation, Lab on a Chip 11 (2011) 1656-1663.

[36] R.J. Umpleby, S.C. Baxter, A.M. Rampey, G.T. Rushton, Y.Z. Chen, K.D. Shimizu, Characterization of the heterogeneous binding site affinity distributions in molecularly imprinted polymers, Journal of Chromatography B: Analytical Technologies in the Biomedical and Life Sciences 804 (2004) 141-149.

[37] G. Sener, L. Uzun, R.I. Say, A. Denizli, Use of molecular imprinted nanoparticles as biorecognition element on surface plasmon resonance sensor, Sensors and Actuators, B: Chemical 160 (2011) 791-799.

[38] R.C. Weast, et al., Handbook of Chemistry and Physics, 53 ed., CRC Press, Cleveland, 1972.

\section{Biographies}

M. Peeters is a PhD student at the Institute for Material Research (IMO) of Hasselt University. She is currently working on the in vivo detection of small molecules based on polymer-type immobilization platforms. She graduated from Eindhoven University of Technology in 2009 with a master degree in Polymer Chemistry.

F.J. Troost is assistant professor at the Department of Internal Medicine, Division of Gastroenterology-Hepatology, Maastricht University Medical Centre, Maastricht, The Netherlands. He obtained his PhD degree in nutrient-gut interactions in humans in 2003, and is senior scientist in several projects, targeting to obtain in-depth information on, and improve gut health with nutritional interventions. The human in vivo intervention studies focus on the effects of nutrition and nutrient metabolites on appetite, mucosal secretions, nutrient sensing and visceral perception.

B. van Grinsven is a $\mathrm{PhD}$ student at the Institute for Material Research (IMO) of Hasselt University. He is currently working on the development of electronic read -out strategies for the detection of single nucleotide polymorphisms in DNA. Before he started his $\mathrm{PhD}$ at Hasselt University he was appointed as a researcher in the R\&D department of TNO (the Netherlands). He graduated from Hasselt University in 2007 with a master degree in bio-electronics and nanotechnology.

F. Horemans graduated from Hasselt University in 2006 with a master degree in bioelectronics and nanotechnology. He obtained his $\mathrm{PhD}$ in 2011 at Hasselt University (Belgium) in the field of molecularly imprinted polymers. Currently, he is working in the group of Biosensors in the Institute for Materials Research (IMO), Hasselt University, Belgium as a post-doc researcher.

J. Alenus is a PhD student at the Institute for Material Research (IMO) of Hasselt University. He is currently working on the development of new biosensor architectures. He graduated from Hasselt University in 2007 with a master degree in bio-electronics and nanotechnology. 
M.S. Murib received his B.Sc. degree in Physics from Lebanese University, Faculty of Science, Hadath, Lebanon, in 2005. He joined the M.Sc. program in Physics at Koç University in Istanbul, Turkey in 2007, as a teaching/research assistant during which he worked with Prof. Ali Serpengüzel. In 2010, he joined the Bios group at IMO, Hasselt University, for his PhD and he is working under the supervision of Prof. Patrick Wagner on bioelectronics and biophotonics sensors.

D. Keszthelyi is a PhD student at the Department of Internal Medicine, Division of Gastroenterology-Hepatology, Maastricht University Medical Center, the Netherlands. He holds an MD degree from the University of Pécs, Hungary. His primary research interest is intestinal serotonin metabolism and the effects of serotonergic modulation on intestinal physiology.

A. Ethirajan graduated Master of Science (2004) in Advanced Materials with the specialization in nanomaterials from Ulm University, Germany. She pursued her PhD (2004-2008) under the guidance of Prof. Dr. Katharina Landfester, Institute of Macromolecular Chemistry and Organic Materials, Ulm University (Germany) and got acquainted with the synthesis of polymer nanoparticles using the miniemulsion technique. Later, she worked in the Max-Planck Institute for Polymer Research, Mainz, Germany for the period 2008-2010 as a post-doc researcher in the research group Physical Chemistry of Polymers. Currently, she is working in the group of Biosensors in the Institute for Materials Research (IMO), Hasselt University, Belgium as a post-doc researcher.
R. Thoelen obtained his PhD in the field of biosensors at Hasselt University in 2008. He is currently an assistant professor at XIOS Hogeschool Limburg.

T.J. Cleij obtained his PhD in 1999 at Utrecht University (The Netherlands) in polymeric materials science. After a stay of three years in the United States as an assistant professor at Louisiana State University, he is currently appointed as a full professor of organic and bio-polymer chemistry at Hasselt University (Belgium) in the Institute for Material Research. The expertise of the Laboratory for Organic and Bio-Polymer Chemistry focuses on the design, synthesis, characterization and application of functional polymeric materials. State of the art facilities for polymer synthesis and characterization are available. He has published over 50 papers on polymeric materials science and engineering.

P. Wagner obtained his PhD in 1994 at Technical University Darmstadt (Germany) in experimental solid state physics with a focus on cuprate superconductors. From 1995 until 2001, he was postdoctoral researcher in the Laboratory of Solid State Physics and Magnetism at Catholic University Leuven (Belgium), where he studied the magneto-transport properties of mixed-valency magnetic oxides. Since 2001, he is a professor of physics at Hasselt University (Belgium) and responsible for the development of label-free readout techniques for DNA-, protein-, and smallmolecule sensors. Patrick Wagner is recipient of a WE Heraeus award, a Marie-Curie Fellowship of the European Union, a Methusalem Grant of the Flemish Government and is past president of the Belgian Physical Society. 